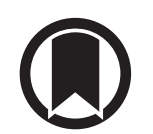

CrossMark

\title{
Lobectomy should remain the first choice for treating early stage nonsmall cell lung cancer
}

\author{
To the Editor:
}

Stereotactic body radiotherapy (SBRT), also known as stereotactic ablative radiotherapy, is characterised as a highly noninvasive precise radiotherapy that is performed by applying an external focused beam guided by high-resolution imaging to target well-defined small tumours [1]. Because of its high local control and survival with acceptable toxicity, SBRT has become the guideline-recommended choice for treating early stage nonsmall cell lung cancer (NSCLC) patients who are not fit for surgery [2]. However, recently, SBRT has also imposed great challenges to surgery in treating operable patients with early stage NSCLC [3]. However, in the absence of completed randomised controlled trials, the currently available evidence for comparing the efficacy of SBRT and surgery for treating early stage NSCLC has all come from observational studies with propensity score-matched analysis [4]. A recent study by DeTiLLon et al. [5] has compared the efficacy of SBRT with video-assisted thoracoscopic (VATS) lobectomy for treating stage I NSCLC in elderly patients by applying propensity score-matched analysis. They included a total of 792 elderly patients $(\geqslant 65$ years old) with clinical stage I NSCLC undergoing SBRT or VATS lobectomy. In the analysis of unmatched cohorts, with significant baseline characteristics imbalance between the two groups, they found that VATS lobectomy yielded significantly better overall survival than SBRT (77 months versus 38 months; $\mathrm{p}<0.001)$. In the analysis of matched cohorts, with well-balanced characteristics between the two groups, VATS lobectomy yielded similar overall survival to SBRT in the first 15 months of follow-up, but significantly better overall survival than SBRT after 15 months of follow-up in both primary and secondary analyses with different matching covariates [5]. Therefore, their study again added to the evidence that patients with clinical stage I NSCLC receiving VATS lobectomy had better overall survival than patients receiving SBRT.

The authors should be complimented for only focusing on the comparison between VATS lobectomy and SBRT by applying propensity score-matched analysis in treating early stage NSCLC, successfully avoiding the bias of different surgical approaches (thoracotomy or VATS) and extents of resection (sublobar or lobectomy), as well as patient selection [5]. In our previous meta-analysis pooling a total of 12 well matched cohort studies involving a total of 13598 patients [4], we also realised the importance of subgrouping surgery into lobectomy and sublobar resection when making comparison between surgery and SBRT. We found that SBRT yielded comparable 3-year survival rate and locoregional control rate to lobectomy but significantly shorter overall survival than lobectomy (hazard ratio $1.68,95 \%$ CI 1.09-2.60; $\mathrm{p}=0.018$ ), whereas SBRT was comparable to sublobar resection in terms of 3-year survival rate and locoregional control rate and even overall survival [4]. Therefore, considering the results from DeTILLON et al. [5] that SBRT yielded comparable overall survival to VATS lobectomy in the first 15 months of follow-up, and our results that SBRT yielded comparable 3-year survival rate and locoregional control rate rate to both lobectomy and sublobar resection, we believe that SBRT may offer good short-term control of early stage NSCLC. However, considering that, even in clinical stage IA NSCLC, the rate of lymph node metastasis was reported to be as high as $28 \%$ [6], and surgery exhibited the advantages of obtaining a definitive tissue diagnosis and accurate nodal evaluation, as well as possible upstaging for adjuvant therapy $[5,7]$, we believe that for long term survival, surgery, especially lobectomy, should still remain the priority in treating early stage NSCLC.

@ERSpublications

Lobectomy should remain the first choice for treating early stage nonsmall cell lung cancer http://bit.ly/2M982Il

Cite this article as: Deng H-Y, Zhou Q. Lobectomy should remain the first choice for treating early stage nonsmall cell lung cancer. Eur Respir J 2019; 54: 1900649 [https://doi.org/10.1183/13993003.00649-2019]. 
Han-Yu Deng and Qinghua Zhou

Lung Cancer Center, West China Hospital, Sichuan University, Chengdu, China.

Correspondence: Qinghua Zhou, Lung Cancer Center, West China Hospital of Sichuan University, No.37 Guoxue Alley, Chengdu 610041, Sichuan, PR China. E-mail: zhouqh135@163.com

Received: March 312019 | Accepted after revision: April 092019

Conflict of interest: None declared.

\section{References}

1 Solda F, Lodge M, Ashley S, et al. Stereotactic radiotherapy (SABR) for the treatment of primary non-small cell lung cancer; systematic review and comparison with a surgical cohort. Radiother Oncol 2013; 109: 1-7.

2 Boily G, Filion E, Rakovich G, et al. Stereotactic ablative radiation therapy for the treatment of early-stage non-small-cell lung cancer: CEPO review and recommendations. J Thorac Oncol 2015; 10: 872-882.

3 Chang JY, Senan S, Paul MA, et al. Stereotactic ablative radiotherapy versus lobectomy for operable stage I non-small-cell lung cancer: a pooled analysis of two randomised trials. Lancet Oncol 2015; 16: 630-637.

4 Deng HY, Wang YC, Ni PZ, et al. Radiotherapy, lobectomy or sublobar resection? A meta-analysis of the choices for treating stage I non-small-cell lung cancer. Eur J Cardiothorac Surg 2017; 51: 203-210.

5 Detillon D, Aarts MJ, De Jaeger K, et al. Video-assisted thoracic lobectomy versus stereotactic body radiotherapy for stage I nonsmall cell lung cancer in elderly patients: a propensity matched comparative analysis. Eur Respir J 2019; 53: 1801561.

6 Zhang D, Chen X, Zhu D, et al. Intrapulmonary lymph node metastasis is common in clinically staged IA adenocarcinoma of the lung. Thorac Cancer 2019; 10: 123-127.

7 Chen H, Louie AV. SABR vs. limited resection for non-small cell lung cancer: are we closer to an answer? Curr Treat Options Oncol 2016; 17: 27. 